\title{
Detección de agentes químicos
}

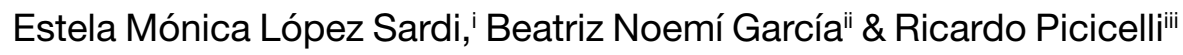

\section{Resumen}

El uso de contaminantes químicos, biológicos, radiológicos y nucleares con fines bélicos se conoce como guerra QBRN. La defensa contra este tipo de agresiones se basa en la detección, monitoreo, neutralización y aislamiento de los agentes.

Actualmente los laboratorios de química analítica más desarrollados están dotados de instrumental sofisticado, utilizado para la detección de compuestos tóxicos industriales y contaminantes ambientales. Estos desarrollos tecnológicos permiten su adaptación para la detección de agentes de guerra química. En el trabajo se presentan los resultados de un análisis de las tecnologías analíticas más avanzadas que pueden ser empleadas en la etapa de detección e identificación de agentes químicos.

Palabras clave: Guerra química. Detección de agentes químicos. Monitoreo de agentes químicos.

Universidad de la Defensa Nacional. UNDEF. Escuela Superior Técnica. Facultad del Ejército. Argentina.

ii Universidad de la Defensa Nacional. UNDEF. Escuela Superior Técnica. Facultad del Ejército. Argentina.

iii Universidad de la Defensa Nacional. UNDEF. Escuela Superior Técnica. Facultad del Ejército. Argentina 


\section{Abstract}

The use of chemical, biological, radiological and nuclear contaminants for war purposes is known as CBRN warfare. The defense against this type of aggressions is based on the detection, monitoring, neutralization and isolation of the agents. Currently the most developed analytical chemistry laboratories are equipped with sophisticated instruments, used for the detection of industrial toxic compounds and environmental contaminants. These technological developments allow their adaptation for the detection of chemical warfare agents. In this paper, we present the results of an analysis of the most advanced analytical technologies that can be used in the detection and identification stage of the defense against CBRN warfare.

Keywords: Chemical warfare. Detection of chemical agents. Monitoring of chemical agents.

Fecha de recepción 10/05/2017 - Fecha de aceptación 04/08/2017 


\section{Introducción}

El ataque con gas sarín del 4 de abril de 2017 a la ciudad de Jan Seijun, en el norte de Siria, dejó un saldo de 83 muertos, 20 de ellos niños, y al menos 560 afectados. (El Mundo, 2017). Este episodio reabrió la discusión internacional sobre las armas químicas. Los últimos antecedentes registrados de este tipo de incidentes son Alepo en marzo de 2013 y Damasco en abril de 2013. (El País, 2013).

El uso de contaminantes químicos, biológicos y nucleares con fines bélicos se conoce como guerra QBN. La lista de sustancias potencialmente peligrosas utilizadas a tal fin es relativamente corta, pero estos agentes, una vez diseminados, tienen la capacidad de causar una gran cantidad de víctimas. (Kortepeter et al. 1999). La defensa contra este tipo de agresiones se basa en la detección, monitoreo, neutralización y aislamiento de estos agentes. Los agentes químicos y biológicos pueden ser dispersados en el aire que respiramos, en el agua que tomamos, o en las superficies con las que tenemos contacto. Los métodos de dispersión pueden ser tan simples como la apertura de un contenedor, el uso de dispositivos de dispersión hogareños (jardinería), o elaborados como la detonación de un explosivo. (Gob. De Canadá, 2008).

Los modernos sistemas misilísticos han incrementado la efectividad del ataque, reduciendo los tiempos para tomar las medidas de protección adecuadas y disminuyendo la cantidad de agente químico requerido para ocasionar el efecto que se busca. (Pietrobelli, 2003).

Las diferencias entre un incidente de agresión química, biológica o nuclear (radiológica) se detallan en la Tabla 1.

Tabla 1. Diferentes tipos de incidentes QBN.

\begin{tabular}{|l|l|l|}
\hline Incidente & Desarrollo de síntomas & Características especiales \\
\hline Químico & $\begin{array}{l}\text { Rápido desarrollo de } \\
\text { síntomas médicos (de } \\
\text { minutos a horas). }\end{array}$ & $\begin{array}{l}\text { Presentan elementos fácilmente observables, } \\
\text { como residuos coloreados, follaje muerto, olor } \\
\text { penetrante y animales o insectos muertos. Las } \\
\text { víctimas se encuentran en la dirección del viento. }\end{array}$ \\
\hline Biológico & $\begin{array}{l}\text { Desarrollo de síntomas } \\
\text { que va de horas a días. }\end{array}$ & $\begin{array}{l}\text { Los agentes biológicos son usualmente incoloros } \\
\text { e inodoros. Debido al tiempo prolongado en el } \\
\text { desarrollo de síntomas, el área afectada por un } \\
\text { incidente biológico puede ser mayor debido al } \\
\text { movimiento de individuos afectados. }\end{array}$ \\
\hline
\end{tabular}

Continúa... 


\begin{tabular}{|l|l|l|}
\hline Radiológico & $\begin{array}{l}\text { El inicio de síntomas, } \\
\text { si los hay, puede tardar } \\
\text { días, semanas o mayor } \\
\text { tiempo }\end{array}$ & $\begin{array}{l}\text { Generalmente, no habrá señales características } \\
\text { porque los materiales radiactivos son } \\
\text { generalmente inodoros e incoloros. Se requiere un } \\
\text { equipo especializado para determinar el tamaño } \\
\text { del área afectada, y si el nivel de la radiactividad } \\
\text { presenta un peligro para la salud inmediato o } \\
\\
\end{array}$ \\
& $\begin{array}{l}\text { a largo plazo. Debido a que la radiactividad no } \\
\text { es perceptible sin el equipo especial, el área } \\
\text { afectada puede ser mayor debido a la migración } \\
\text { de individuos contaminados. }\end{array}$ \\
\hline
\end{tabular}

La biodefensa se basa en dos Tratados: el Protocolo para la Prohibición del Empleo en Guerra de Gases Asfixiantes, Venenosos y Otros Métodos Bacteriológicos de Guerra (Protocolo de Ginebra de 1925) y la Convención sobre la Prohibición de Desarrollo, Producción y Almacenamiento de las Armas Bacteriológicas (Biológicas) y Toxínicas y Sobre su Destrucción de 1972 (conocido por la sigla CWC), que es el más completo y se pretendía definitivo. Ninguno de los Tratados incluye sistema alguno de verificación. Los Estados-Partes, conscientes de su necesidad, celebraron -hasta el año 2002- múltiples sesiones de trabajo para acordar un Protocolo de Verificación que no se pudo conseguir debido a diversas circunstancias de la geopolítica mundial. Consecuentemente, la Convención de Prohibición de las Armas Biológicas sigue siendo un mero instrumento jurídico testimonial y la indefensión de la humanidad una evidencia. (Fuente Sánchez, 2012).

\section{Los agentes químicos}

Según el artículo II de la CWC, se denomina arma química a: 1) las sustancias químicas tóxicas o sus precursores, salvo cuando se destinen a fines no prohibidos por la presente Convención, siempre que los tipos y cantidades de que se trate sean compatibles con esos fines; 2) las municiones o dispositivos destinados de modo expreso a causar la muerte o lesiones mediante las propiedades tóxicas de las sustancias especificadas en el apartado 1 que liberen el empleo de esas municiones o dispositivos; o 3) cualquier equipo destinado de modo expreso a ser utilizado directamente en relación con el empleo de las municiones o dispositivos especificados en el apartado 2. Según la CWC, los elementos descritos en 1, 2 y 3 deben estar presentes para constituir un arma química. Según la CWC, una sustancia química tóxica es toda sustancia química que, por su acción química sobre los procesos vitales, pueda causar la muerte, la incapacidad temporal o lesiones permanentes a seres humanos o animales. Un precursor es cualquier reactivo 
químico que intervenga en cualquier fase de la producción por cualquier método de una sustancia química tóxica. (Cruz Roja, 1994).

\section{Propiedades físico químicas de los agentes de guerra}

Las propiedades físico-químicas a tener en cuenta en un agente de guerra químico son:

Densidad de vapor absoluta: cociente entre su masa molar y el volumen molar en CNPT $\left(22,4 \mathrm{dm}^{3)}\right.$.

Densidad de vapor relativa: cociente entre la densidad de un gas y la densidad del aire en iguales condiciones de presión y temperatura. Los gases más densos que el aire tienden a permanecer a nivel del suelo. Prácticamente todos los agentes químicos gaseosos son más densos que el aire, a excepción del cianuro de hidrógeno, el amoníaco y el monóxido de carbono.

Presión de vapor: cantidad de vapor necesario para saturar el aire. Altas presiones de vapor indican gran volatilidad y bajas presiones de vapor indican baja volatilidad y por lo tanto mayor persistencia.

Volatilidad: Cantidad máxima de un compuesto químico vaporizado por metro cúbico de aire a una cierta temperatura. La volatilidad de estos agentes supera la concentración IDLH (concentración inmediatamente peligrosa para la vida o la salud).

Toxicidad: Cantidad de sustancia necesaria para producir efectos perjudiciales sobre un ser vivo.

Persistencia: Tiempo durante el cual el agente mantiene su peligrosidad.

\section{Clasificación de los agentes químicos:}

Los agentes químicos se agrupan en categorías según los efectos fisiológicos que producen en los seres vivos. (Matatagui cruz, 2012; Martínez Pons, 2006). Se conocen:

- Agentes neumotóxicos o sofocantes, dañan los pulmones, dificultan o impiden la respiración.

- Agentes vesicantes, causan ampollas y graves lesiones en la dermis.

- Agentes nerviosos o neurotóxicos, atacan al sistema nervioso mediante la inhibición de neurotransmisores.

- Agentes sanguíneos, impiden la oxigenación de la sangre.

- Agentes incapacitantes, provocan parálisis, confusión, alucinaciones y otros efectos. 
- Agentes irritantes, como los gases lacrimógenos.

- Agentes biológicos, capaces de producir enfermedades letales, como ántrax, muermo, tifus, brucelosis y carbunco, entre muchos otros.

\section{Principales agentes químicos}

\section{Agentes sofocantes}

Sustancias que causan daño físico a los pulmones. La exposición es a través de la inhalación, y en los casos más graves las membranas se hinchan y los pulmones se llenan de líquido (edema pulmonar). La muerte es por falta de oxígeno. El Cloro, Fosgeno (CG), y Cloropicrina (PS) son los principales agentes sofocantes empleados que se caracterizan porque sus síntomas no aparecen hasta varias horas tras su exposición. En la Imagen 1 se detalla la estructura química de estos agentes.

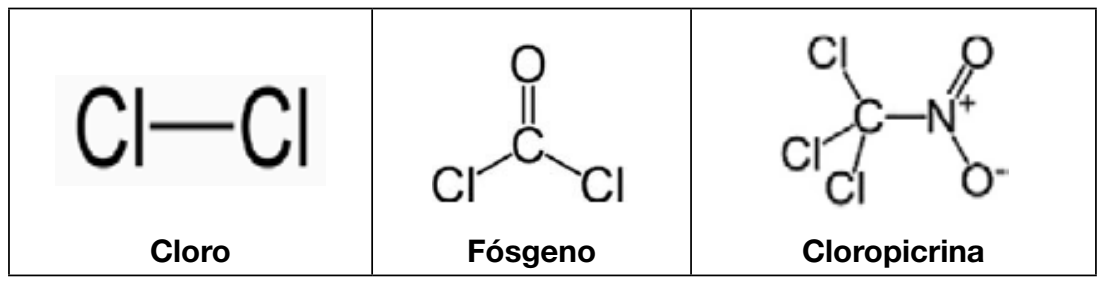

Imagen 1. Estructura de agentes neumotóxicos.

\section{Agentes vesicantes}

Sustancias que causan quemaduras profundas en la piel que pueden destruir los tejidos internos. La exposición puede ser por contacto de líquido o vapor con cualquier tejido expuesto (ojos, piel o pulmones). Los principales agentes vesicantes son: Mostaza Azufrada o Iperita (HD), Mostaza Nitrogenada (HN) y Lewisita (L). Sus estructuras se observan en la Imagen 2.

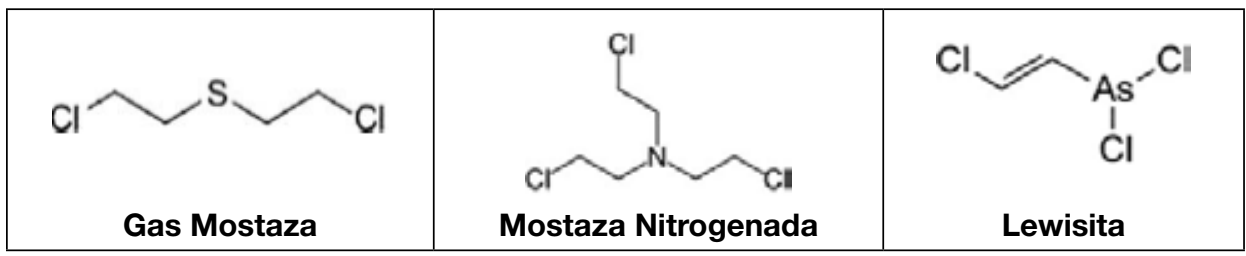

Imagen 2. Estructura de agentes vesicantes. 


\section{Agentes nerviosos}

Son sustancias organofosforadas que actúan en el Sistema Nervioso Central bloqueando la acción de la Acetilcolinesteresa, enzima responsable del impulso nervioso. En condiciones normales son líquidos incoloros y su potencial como arma se basa en el átomo de fósforo, cuyos enlaces son fácilmente hidrolizados por compuestos como el agua o iones álcali. Hay dos familias de gases nerviosos que se utilizan principalmente para fines militares, la serie $\mathrm{G}$, que actúa por inhalación y los de la la serie $\mathrm{V}$ que actúan principalmente a través de la piel. Los agentes nerviosos pueden ser degradados por las sustancias que se encuentran en el aire, por lo que persisten pocos días. En agua y suelo húmedo son rápidamente hidrolizados y sólo pequeñas cantidades se evaporarán. A través del suelo pueden contaminar el agua subterránea pero nunca se acumulan en la cadena alimentaria. Sus estructuras se pueden observar en la Imagen 3.

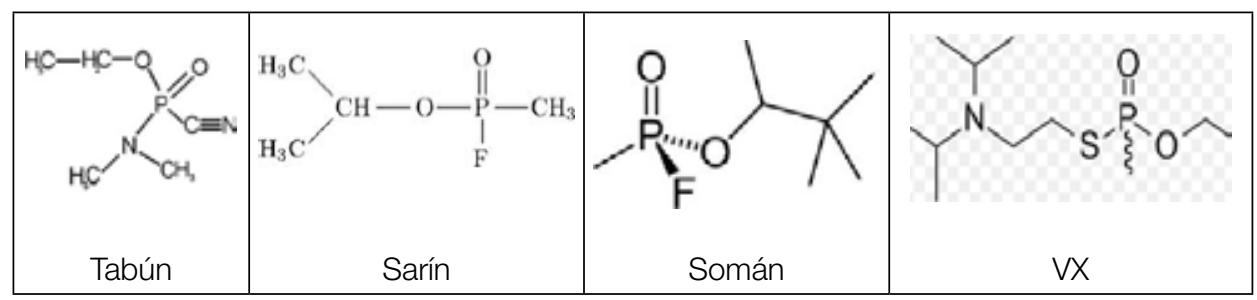

Imagen 3. Estructura de agentes nerviosos.

\section{Agentes sanguíneos}

Los agentes sanguíneos son sustancias que provocan daños por interferencia en la respiración celular (intercambio de $\mathrm{O} 2$ y CO2 entre la sangre y los tejidos). El Cianuro de Hidrógeno (AC) y el Cloruro de Cianógeno (CK) son dos agentes sanguíneos utilizados como armas químicas. Los síntomas más relevantes son: dolor al respirar, cefalea, insensibilidad, ataque y coma. Sus estructuras se visualizan en la Imagen 4.

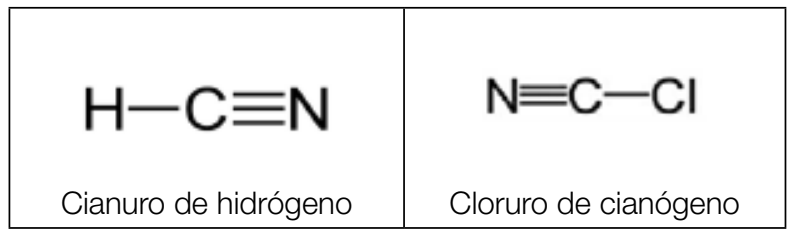

Imagen 4. Estructura de agentes sanguíneos. 


\section{Agentes incapacitantes}

Los agentes incapacitantes son todas aquellas sustancias que provocan incapacidad mental o física temporal. Se distinguen por la duración de la incapacidad causada:

- Los productos químicos que causan invalidez durante algún tiempo mayor al de la exposición, como es el caso de la mayor parte de los agentes irritantes utilizados por la policía para el control de disturbios. A este grupo se le conoce como neutralizantes, irritantes o agentes hostigadores.

- Aquellos productos químicos que causan incapacidad durante un periodo bastante mayor al de la exposición. A este grupo se les conoce comúnmente como calmantes.

En ambas categorías el tiempo de inicio de la incapacidad es también un determinante importante de su utilidad. Los agentes neutralizantes producen irritación fundamentalmente en los ojos y el sistema respiratorio, como los gases lacrimógenos CS y CN, cuyas estructuras vemos en la Imagen 5.

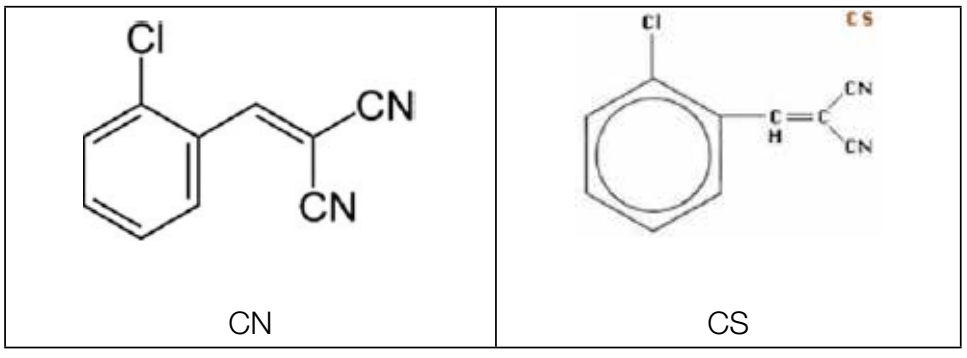

Imagen 5. Estructura de agentes neutralizantes.

Entre los agentes calmantes el más utilizado es el BZ, cuyos efectos psíquicos y psicológicos, como graves dificultades motoras y confusión pueden durar hasta 90 horas. En la Imagen 6 se observa su estructura química.

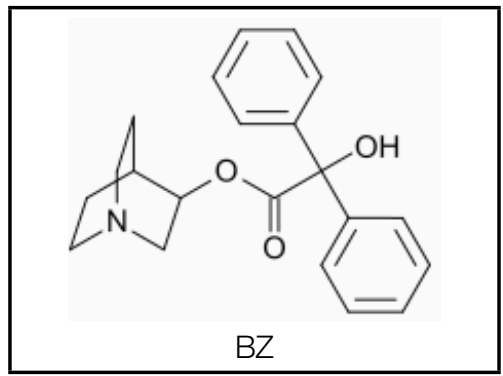

Imagen 6. Estructura del BZ. 


\section{Equipos para detección de agentes químicos}

Los factores relacionados con la capacidad de detección de un equipo son:

Selectividad: capacidad del selector de responder exclusivamente a los químicos similares a los que se encuentran en su librería.

Sensibilidad: esta aumenta cuanto más grande sea el cambio de intensidad de la señal frente a un pequeño cambio en la concentración del analito.

Limite de detección: menor concentración a la que es posible detectar al analito con un cierto grado de confianza.

Capacidad de análisis cuantitativo: la capacidad de proporcionar resultados cuantitativos confiables disminuye en el trabajo de campo respecto al trabajo en el laboratorio debido a las condiciones aleatorias del ambiente externo. Sin embargo suele ser suficiente que el equipo pueda dar en campo una respuesta cualicuantitativa que permita establecer que el analito supero los límites a partir de los cuales se convierte en amenaza.

Tasa de falsas alarmas (positivas o negativas): este problema se puede minimizar usando simultáneamente más de un sistema de detección.

Tiempo de respuesta: frente a un compuesto una vez iniciado el proceso de detección. Suele aumentar frente a la disminución de la concentración.

Otros factores a tener en cuenta son el tiempo de arranque y calentániento, los requerimientos de calibración, la dependencia de otros materiales como baterías y filtros, su portabilidad y la facilidad de uso. (Acuña Benito et al, 2011).

\section{Equipos portátiles}

\section{Equipo de detección colorimétrica}

Analizan los cambios de color producidos durante la reacción química entre un reactivo y el analito considerado. El cambio puede apreciarse a simple vista o mediante un lector portátil. Suelen utilizarse tiras reactivas. El cambio de color indica la presencia del contaminante. La intensidad del color o la longitud de la banda coloreada indican su concentración. En la Tabla 2 se citan los principales sistemas de detección colorimétrica: 
Tabla 2. Sistemas de detección colorimétrica.

\begin{tabular}{|l|l|}
\hline Tubos colorimétricos & $\begin{array}{l}\text { Kits desechables para detección en el aire } \\
\text { (fase vapor) de agentes de guerra nerviosos } \\
\text { vesicantes, sanguíneos y sofocantes. }\end{array}$ \\
\hline $\begin{array}{l}\text { Papeles de Detección M8, M9 y 3-way } \\
\text { Tradeways Ltd. }\end{array}$ & $\begin{array}{l}\text { Detección de agentes de guerra nerviosos y } \\
\text { vesicantes en fase líquida. }\end{array}$ \\
\hline M256A1 Deector Kit & $\begin{array}{l}\text { Agentes nerviosos sanguíneos y vesicantes en } \\
\text { fase líquida y/o vapor. }\end{array}$ \\
\hline
\end{tabular}

\section{Espectroscopía por movilidad iónica}

Esta técnica, conocida como IMS, separa y detecta, a temperatura ambiente y presión atmosférica, iones en función de su velocidad al atravesar un gas portador bajo la influencia de un campo eléctrico. La muestra es ionizada generalmente mediante el uso de isótopos radiactivos, los iones forman clusters cargados atraídos por un pulso de campo eléctrico de signo contrario. La variable de detección es el tiempo de vuelo o tiempo que tardan en llegar al detector, el cual dependerá de su masa y tamaño.

Existe multiplicidad de equipos portátiles que aplican esta tecnología, entre los que podemos mencionar Brucker Daltonics, Smith Detection, y Naval Detection Systems. Los equipos permiten la detección de gases nerviosos, vesicantes, sanguíneos y sofocantes, con muy bajos tiempos de respuesta.

\section{Espectroscopía por movilidad diferencial}

Esta tecnología representa una mejora respecto de IMS y aunque está aún en fase de investigación ya existe un equipo comercial, de General Dynamics Armament and Technical Products, el cual permite detectar agentes de guerra química en fase vapor. El fundamento de esta técnica consiste en que los iones, moléculas o partículas cargadas que se quieren analizar son arrastrados a través de una región ocupada por un fluido debido a la presencia de un campo eléctrico. Estos iones o partículas cargadas son desviados por el desplazamiento del fluido en una corriente perpendicular al campo eléctrico. La mayor o menor separación de su trayectoria respecto a las líneas de campo eléctrico depende de la movilidad eléctrica de la partícula. [6].

\section{Fotometría de llama}

En condiciones normales los electrones de los átomos permanecen en las órbitas que hacen que la energía total del sistema sea mínima. Pero frente a una excitación 
(por medio de luz, calor o descarga eléctrica) los electrones sufren una transición hacia un orbital de mayor energía. Decimos que el átomo se encuentra en "estado electrónicamente excitado". Cuando cesa la excitación, los electrones tienden a volver a su estado normal, emitiendo una cantidad de energía igual a la diferencia entre el estado excitado y el estado normal. La radiación devuelta corresponde a longitudes de onda específicas, de acuerdo con las transiciones permitidas para cada elemento químico, lo que constituye su espectro de emisión característico. La intensidad medida para cada una de las longitudes de onda se encuentra en relación directa con la cantidad de átomos excitados (concentración). (UBA, 2017). El detector portátil AP2C/AP4C de la empresa Proengin es el más difundido entre los que emplean esta tecnología, permitiendo detectar compuestos que contienen fósforo y azufre en fase vapor, gotas, polvo, líquidos, sólidos e incluso en muestras congeladas.

\section{Detectores de fotoionización}

Los detectores de fotoionización (PID) usan una fuente de luz ultravioleta de alta energía para ionizar sustancias orgánicas volátiles en muy bajas concentraciones (partes por millón, ppm). Las sustancias ionizadas son conducidas al detector a través de un gradiente eléctrico. El potencial de ionización es específico para cualquier compuesto o especie atómica y proporcional a la concentración de la sustancia, de forma que el detector mide esta corriente y muestra la concentración de la sustancias en partes por millón (ppm) o por billón (ppb). Esta tecnología es muy utilizada para la detección de compuestos orgánicos volátiles. La PID no es selectiva, de forma que cualquier molécula con un potencial de ionización bajo puede ser ionizada con una fuente de ionización de alta energía. Las fuentes UV pueden mejorar la selectividad en compuestos con altos potenciales de ionización. [6]. La empresa RAE Systems ofrece equipos portátiles que incorporan esta tecnología.

\section{Detectores de onda acústica superficial}

Los sensores de onda acústica superficial, SAW, se llaman así porque su mecanismo de detección es una onda mecánica o acústica. Cuando esta onda acústica se propaga a través o sobre la superficie de un material, cualquier cambio en las características del curso de propagación afecta a la velocidad y/o amplitud de la onda. Los cambios en la velocidad pueden ser monitorizados a través de la medida de la frecuencia o las características de fase del sensor, de forma que por correlación finalmente se obtiene una cantidad física medible. La onda es generada por material piezoeléctrico. [6]. Se comercializan distintos sensores portátiles de trazas por tecnología SAW aplicables a detectar agentes de guerra químicos nerviosos, como el HAZMADCAD Plus de MSA, 
CW SentryTM 3G de Microsensors Systems Inc., ChemSentry 150G de RAE Systems. Tecnologías combinadas

Las empresas Environics y Airsense Analitics ofrecen detectores portátiles que combinan dos o más de estas tecnologías en un mismo dispositivo. Generalmente se combinan IMS, fotoionizador, célula electroquímica y sensores de óxido de metal o semiconductores.

\section{Equipos a distancia}

Llamados también equipos stand-off, se dividen en pasivos y activos.

Los equipos pasivos graban el espectro infrarrojo emitido o absorbido por los CWA, preferentemente en fase vapor, en función del distinto gradiente térmico existente entre los mismos y el fondo atmosférico. Como desventaja no pueden detectar con precisión la fuente de la señal y su ventaja es que son difícilmente detectables.

Los equipos activos realizan un análisis atmosférico mediante un láser remoto como fuente de excitación. Permiten detectar matemáticamente la fuente de la señal pero su ubicación es fácilmente detectable. Detectan mejor los CWA en fase aerosol.

\section{Detección por espectrofotometría infrarroja}

El espectro es originado por el alargamiento vibracional y flexión de los enlaces químicos en los distintos grupos funcionales que constituyen una molécula orgánica. El espectro infrarrojo es una huella dactilar del compuesto, ya que no existen dos compuestos distintos con espectro IR idéntico. Las empresas Bertin Technologies y Avin Sensors ofrecen equipos que aplican esta tecnología.

\section{Espectroscopía Infrarroja por Transformada de Fourier}

Tecnología conocida como FTIR. La transformada de Fourier aumenta la sensibilidad del sistema por tratarse de un algoritmo que permite la descomposición de una señal en componentes de frecuencias distintas y medibles. Existen múltiples facilidades que utilizan esta técnica, entre ellas las de las empresas General Dynamics Armament and Technical Products y Bruker Daltonics.

\section{Espectroscopía de Absorción infrarroja con láser de cascada cuántica}

El láser de cascada cuántica aumenta la sensibilidad de los espectrómetros al permitir la emisión de luz en una amplia gama del espectro electromagnético usando 
una misma combinación de materiales en la construcción del láser. Esto se logra mediante una estructura cristalina que alterna hasta 1000 capas de distinto espesor entre dos diversos semiconductores, permitiendo variar la longitud de onda dentro de una inmensa gama. Funciona muy bien en el IR mediano. El equipo LaserScan de Block Engineering permite detectar CWA y explosivos tanto en fase vapor como líquida e incluso altamente adsorbidos sobre superficies.

\section{Láser $\mathrm{CO}_{2}$ DIAL (Differential absorption lidar)}

Esta tecnología realiza las mediciones usando dos longitudes de onda denominadas on-line (ION) and off-line (IOFF). La ION es la longitud de onda absorbida por el gas a ser medido, la IOFF es una longitud de onda de referencia no absorbida por el gas. Ambas longitudes de onda deben ser cercanas, para evitar líneas de absorción de moléculas interferentes. (Robinson et al., 2014). Ambos pulsos láser son emitidos, atraviesan la nube química, se reflejan en alguna característica del terreno y son nuevamente recibidos por el equipo. La presencia del agente se traduce en la atenuación diferencial de la señal ION, que permite calcular la concentración del agente en la columna atmosférica atravesada. El equipo DDCWA de la empresa VOP TRENCIN incorpora esta tecnología.

\section{Equipos de identificación de armas químicas}

Una vez detectado el agente y estimada su naturaleza, se requiere de una identificación inequívoca del CWA. Para ello se utilizan distintas tecnologías de la química analítica.

\section{Tandem cromatógrafo gaseoso- espectrómetro de masas}

Este acoplamiento es posible porque el caudal de un CG es lo suficientemente bajo como para ser introducido en la cámara de ionización de un EM. A la salida del CG los distintos componentes de la mezcla, ya identificados por su tiempo de retención salen separados e ingresan en el EM, donde son fragmentados e identificados por su relación carga/masa. La identificación coincidente de los compuestos en ambas librerías informáticas potencia enormemente la certeza en los resultados.

Los equipos GRIFFIN 460 de la empresa Flir, HAPSITE de Inficon, MM2 Mobile Mass Spectrometer de Bruker Daltonics combinan estas dos tecnologías. 


\section{Espectroscopía Raman}

El efecto Raman se produce cuando un fotón de un haz de luz monocromático incide sobre una molécula e interacciona sobre la nube electrónica de sus enlaces. La dispersión observada cuando el sistema vuelve al estado inicial se conoce como Raman - Stokes si el punto de partida fue una molécula en su estado fundamental, o Raman - Anti Stokes si el proceso se inició sobre una molécula en estado vibracional excitado. El patrón de frecuencias de desplazamiento está determinado por los estados vibracionales y rotacionales propios de la muestra bajo estudio. El haz de luz monocromática empleado puede estar en el rango visible, IR cercano o UV cercano. (Cambridge, 2007)

Esta técnica no implica preparación previa del material ni su destrucción. Equipos como el responder RCI de Smiths Detection, ReporteR de Airsense Analytics y First Defender de Ahura utilizan esta tecnología. [6]

\section{Conclusiones}

La integración en red de tecnologías analíticas desarrolladas para su empleo en otros campos, como la química industrial y la química ambiental, permite el desarrollo de equipamiento altamente eficaz para la detección e identificación de CWA. El avance de la tecnología permite disminuir la tasa de falsas alarmas y optimizar los tiempos de respuesta frente a este tipo de amenazas. La implementación de tecnología actualmente en fase de desarrollo como sensores miniaturizados de nanotubos de carbono permitirá grandes avances en la detección e identificación de CWA.

\section{Bibliografía}

Acuña Benito,A.; Fernández, A.; Bueno, I. et al.; (2011) Detección e identificación de agentes de guerra química: estado de arte y tendencia futura. Sistema de Observación y Prospectiva Tecnológica (SOPT), Subdirección General de Tecnología y Centros, de la Dirección General de Armamento y Material, Ministerio de Defensa, España. Imprenta del Ministerio de Defensa. Consultado el 20 de marzo de 2017 en: http://www.tecnologiaeinnovacion.defensa.gob.es/ Lists/Publicaciones/Attachments/11/monografia_sopt_11.pdf

Airsense. Consultado el 20 de marzo de 2017 en: http://www.airsense.com

Bertin Technologies. Consultado el 20 de marzo de 2017 en: http://www.bertin.fr 
Block Engineering. Consultado el 20 de marzo de 2017 en: http://www.blockeng.com Bruker Daltonics. Consultado el 20 de marzo de 2017 en: https://www.bruker.com

Cátedra de física. UBA. (s.f.). Fundamentos espectroscopía. Consultado el 1 de abril de 2017 en: http://virtual.ffyb.uba.ar/file.php/29/M4/FUND_M4_ ESPECTROSCOPIA.pdf

Convención sobre la prohibición del desarrollo, la producción y el almacenamiento de armas bacteriológicas (biológicas) y Toxínicas y sobre su destrucción. (1972). Tomado del Manual del Movimiento Internacional de la Cruz Roja y la Medialuna Roja, 13a edición, 1994. Consultado el 20 de marzo de 2017 en: https://www.icrc.org/spa/resources/documents/misc/treaty-1972bacteriological-weapons-convention-5tdm6y.htm

El Mundo. (2017). Un ataque químico mata al menos 83 personas en Siria. Edición del 7 de abril de 2017. Consultado el 7 de abril de 2017 en: http:// www.elmundo.es/internacional/2017/04/04/58e35091e5fdeaef348b4573.html

El País. (2013). El ataque químico que viví en Siria. Edición del 27 de mayo de 2013, consultada el 7 de abril de 2017 en: http://internacional.elpais.com/ internacional/2013/05/27/actualidad/1369644094_374656.html

Environics. Consultado el 20 de marzo de 2017 en: http://www.environics.com

Flir. Consultado el 20 de marzo de 2017 en: http://www.flir.com

Fuente Sánchez, J.M. (2012). La defensa biológica: sus grandes carencias. Dialnet. Universidadde La Rioja. España. Consultado el20 de marzo de $2017 \mathrm{en}$ :file://C:/ Users/Monica/Desktop/Descargas/Dialnet-LaDefensaBiologica-4198963.pdf

Gobierno de Canadá. (2008) Uso terrorista/criminal de agentes químicos/ biológicos/radiológicos. Consultado el 20 de marzo de 2017 en: http:// wwwapps.tc.gc.ca/saf-sec-sur/3/gre/gre/criminal.aspx

Inficon. Consultado el 20 de marzo de 2017 en: http://www.inficon.com

Infobae. (2017) El video que muestra los inhumanos efectos de los ataques con gas sarín en Siria. Edición del 4 de abril de 2017. Consultado el 4 de abril de 2017 en: http://www.infobae.com/america/mundo/2017/04/04/el-video-quemuestra-los-inhumanos-efectos-de-los-ataques-con-gas-sarin-en-siria/

Kortepeter, M.G.; Parker, G.W. (1999). Potential biological weapon threats, U.S. Army Medical Research Institute of Infectious Diseases, Fort Detrick, Maryland, USA. Emerging Infectious Diseases, Vol. 5, No. 4, July.August 1999, p 523-527. 
Martínez Pons, J. A.; (2006). Armas químicas: que son y como actúan. An. Quim. 2006, 102(1), 55-64. Real Sociedad Española de Química.

Matatagui Cruz, D. (2012). Sensores másicos para la detección de agentes de guerra química y biológica. Tesis doctoral. Universidad Autónoma de Madrid. Consultado el 30 de abril de 2017 en: https:/dialnet.unirioja.es/servlet/ tesis? codigo $=34322$

Pietrobelli, N. G. (2003). Diseño del elemento de defensa QBN a nivel Gran Unidad de Combate para dar una correcta y oportuna respuesta ante los nuevos escenarios actuales. Trabajo final de licenciatura. CEFA DIGITAL. Consultado el 20 de marzo de 2017 en: http://www.cefadigital.edu.ar/ handle $/ 123456789 / 550$ ?mode $=$ full

Proengin. Consultado el 20 de marzo de 2017 en: http://www.proengin.com/ chemical-detection/ap2c/

Rae Systems. Consultado el 20 de marzo de 2017 en: http://www.raesystems.com

Robinson, I., Jack, J. W., Rae, C. F., \& Moncrieff, J. B. (2014, October). Development of a laser for differential absorption lidar measurement of atmospheric carbon dioxide. In SPIE Remote Sensing (pp. 92460U-92460U). International Society for Optics and Photonics. Consultado el 2/4/17 en: https://scholar.google.com/ scholar?hl=es\&q=Development + of $+\mathrm{a}+$ laser + for + differential + absorption+lida $\mathrm{r}+$ measurement + of + atmospheric + carbon + dioxide $\& b t n G=\& l r=$

Smith Detection. Consultado el 20 de marzo de 2017 en: https://www. smithsdetection.com

Thermo Fisher. Consultado el 20 de marzo de 2017 en: www.thermofisher.com/

University of Cambridge. Teaching and Learning Packages. (2007). Raman Spestroscopy. Consultado el 2 de abril de 2017 en: https://www.doitpoms. ac.uk/tlplib/raman/index.php 